\title{
Treatment of Infiltrative Basal Cell Carcinomas by inhibiting the Fibroblast Growth Factor (FGF)-Signal Transducer and Activator of Transcription (STAT)-3 Signalling Pathways
}

\author{
Pedro Cuevas $^{1 *}$, Javier Angulo ${ }^{1}$, Adrián Cuevas-Bourdier², Irene Salgüero ${ }^{3}$ and Guillermo Giménez-Gallego ${ }^{4}$ \\ ${ }^{1}$ Departamento de Investigación. Servicio de Histología \\ ${ }^{2}$ Servicio de Anatomía Patológica \\ ${ }^{3}$ Servicio de Dermatología Hospital Universitario Ramón y Cajal, IRYCIS, E-28034-Madrid-Spain \\ ${ }^{4}$ Departamento de Estructura y Función de Proteínas. Centro de Investigaciones Biológicas Consejo Superior de Investigaciones Científicas. (CSIC), Madrid
}

\begin{abstract}
Over-expression of FGF/STAT-3 signalling pathways or aberrant regulation of their activities has been implicated in many forms of human malignancies, including skin neoplasms. Therefore, targeting FGF/STAT-3 signalling pathways represents an attractive strategy for development of basal cell carcinoma (BCC) treatment by simultaneously inhibiting tumor cell growth and survival, and also tumor angiogenesis. Here, we describe the efficacy of a safe, potent and selective FGF inhibitor -dobesilate- in BCC patients. Anti-tumor effect of dobesilate correlates with promotion of apoptosis, and inhibition of both cell proliferation and angiogenesis. Our data support dobesilate as an agent for chemoprevention and therapy of skin cancers by inhibiting FGF/STAT-3 oncogenic signalling axis.
\end{abstract}

Keywords: Basal cell carcinoma; Fibroblast growth factor (FGF); Signal transducer and activator of transcription (STAT)-3; Dobesilate

\section{Introduction}

The most important risk factor for skin cancer is chronic exposure to UV radiation in sunlight [1]. The UV light spectrum, which spans between visible light and X-rays, is divided conventionally into three major categories: UVA (315-400 nm) UVB (280-315 nm), and UVC (190-280 nm). Because of the stratosphere filtering effect, the UVB component of sunlight is the most prominent and ubiquitous carcinogenic light component of our natural environment. Basal cell carcinoma (BCC) is the most common skin cancer. UV irradiation is considered to be a major etiological factor for pathogenesis of BCC [2]. Interestingly, fibroblast growth factor (FGF) was secreted after UV-B stimulation [3,4] and was overexpressed in skin neoplasms [5], suggesting that FGF signals have a role in neoplastic alteration of the skin [3]. Recently, it has been reported that FGF promotes activation of signal transducer and activator of transcription (STAT-3) [4,6]. The STAT family of proteins consists of seven members: STAT-14, STAT-5a, STAT-5b; and STAT-6 $[7,8]$. STAT plays an important role in gene expression in cellular responses to stimulation by growth factors and cytokines [9]. Following ligand-receptor interaction, STAT becomes tyrosine-phosphorylated, which induces STAT to homo-or heterodimerize, and to subsequently enter into the nucleus where it recognizes specific gene promoters. Accumulating evidence supports a critical role for STAT-3 in oncogenesis [10]. Constitutive activation of STAT-3 signalling contributes to oncogenesis by preventing apoptosis and enhancing cell proliferation $[10,11]$. Furthermore there is considerable evidence that overexpression of STAT-3 correlates with invasion and metastasis in skin cancers [12,13] through induction of genes encoding matrix metaloproteases [14]. In addition, it has been reported that activation of STAT-3 is linked to angiogenesis enhancement [15] a key biological process in tumor growth and metastasis [16]. More precisely the FGF-STAT-3 signalling pathway is known to play a key role in BCC growth, survival and angiogenesis [17], thereby serving as a potential therapeutic target and marker of successful treatments of BCC.
Although low-molecular weight therapeutic agents as G3139, or oblimersen sodium (Genasense Genta Pharmaceuticals Inc.) against cancer [18] seem attractive because they share excellent molecular properties in terms of stability and bioavailability [19], its clinical use was concomitantly associated with toxicities [20]. Using structurebased screening procedures we have previously identified several compounds that show antitumoral and antiangiogenic activities both in vivo and in vitro [21-23]. Recently we have reported that dobesilate which has been used for many years as a safe vasculoprotective agent [24] is a powerfull inhibitor of FGF-driven neovascularization, cell proliferation, cell migration and apoptosis [25]. Furthermore, dobesilate depresses the activated levels of STAT-3 in glioma cells [26]. Herein we report evidences that dobesilate is clinically efficient in the treatment of infiltrative BCC, and that its application dampens the levels of activated STAT-3, promotes apoptosis, and attenuates cell proliferation and angiogenesis.

\section{Material and Methods}

\section{Patients and treatment}

Two patients with infiltrative BCC in the face were enrolled for the study approved by the Local Ethics Committee. After given their informed consent, patients were treated with a $2.5 \%$ 2,5-dihydroxyphenyl sulfonate cream (Dobesilate, Merck). Topical

*Corresponding authors: Dr. Pedro Cuevas, Servicio de Histología, Departamento de Investigación, IRYCIS, Hospital Universitario Ramón y Cajal, Ctra. de Colmenar km.9.100, E2034-Madrid-Spain, E-mail: pedro.cuevas@hrc.es

Received September 23, 2011; Accepted November 05, 2011; Published November 07, 2011

Citation: Cuevas P, Angulo J, Cuevas-Bourdier A, Salgüero I, Giménez-Gallego G (2011) Treatment of Infiltrative Basal Cell Carcinomas by inhibiting the Fibroblas Growth Factor (FGF)-Signal Transducer and Activator of Transcription (STAT)-3 Signalling Pathways. J Cancer Sci Ther S3:003. doi:10.4172/1948-5956.S3-003

Copyright: (C) 2011 Cuevas P, et al. This is an open-access article distributed under the terms of the Creative Commons Attribution License, which permits unrestricted use, distribution, and reproduction in any medium, provided the original author and source are credited. 
Citation: Cuevas P, Angulo J, Cuevas-Bourdier A, Salgüero I, Giménez-Gallego G (2011) Treatment of Infiltrative Basal Cell Carcinomas by inhibiting the Fibroblast Growth Factor (FGF)-Signal Transducer and Activator of Transcription (STAT)-3 Signalling Pathways. J Cancer Sci Ther S3:003. doi:10.4172/1948-5956.S3-003

Page 2 of 5

treatment was self-applied by the patients twice a day on BCC lesions. Treatment was continued until a complete clinical clearance of the tumor (3 months). Biopsy punches $(2 \mathrm{~mm})$ were performed before and at the end of treatment. Dobesilate cream was prepared at the Pharmacy Department of the Hospital Universitario Ramón y Cajal in Madrid.

\section{Histological studies}

Histopathological confirmation of the diagnosis of BCC was performed in a blinded fashion in deparaffined sections obtained from pre- and post-treatment skin specimens. For paraffin embedding, biopsies were dehydrated in increasing concentrations of alcohol to xylene, followed by changes of hot paraffin wax and embedding biopsies were cut at a thickness of $5 \mu \mathrm{m}$ and dried overnight at $37^{\circ} \mathrm{C}$. Sections were deparaffinized in xylene followed by decreasing concentrations of alcohol (100-70\%) and rinsed in distilled water. Deparaffinized tissue sections were used for routine histology, terminal 2'-deoxyuridine5 'triphosphate (dUTP) nick-end-labelling (TUNEL) staining and immunohistochemistry. For standard histology sections were stained with Mayer's haematoxylin and eosin to determine cellularity and tissue morphology.

\section{TUNEL staining}

For analysis of programmed cell death, we used the in situ detection of apoptosis by TUNEL assay. After permeabilization with $0.1 \%$ Triton X-100, terminal deoxynucleotidyl transferase (TdT) enzyme and dUTP conjugated to biotin were added to slides, according to the manufacturer's specifications (Boehringer Mannheim, Germany). Slides were then incubated with an avidin-biotinylated horseradish peroxidase complex (ABC). Diaminobenzidine (DAB) (Sigma, St. Louis, $\mathrm{MO}$ ) at $0.005 \%$ was used as chromogen. For positive controls, slides were treated with DNase $(1 \mu \mathrm{g} / \mathrm{ml})$. Negative controls included omission of either dUTP, TdT or avidin-biotinylated horseradish peroxidase complex. All sections were then counterstained with Mayer's haematoxylin to obtain a nuclear image of nonapoptotic cells.

\section{Immunohistochemical staining}

To assess potential mechanisms of action of dobesilate, sections were immunohistochemically characterized using antibodies to $\mathrm{CD}^{+}{ }^{+}$(1:100, Dako, Denmark), Ki67 (1:100, Dako, Denmark) and phosphorylated STAT-3 (1:50; Santa Cruz Biotechnology, Santa Cruz, $\mathrm{CA})$. For antigen retrieval, the sections were micro waved twice for $15 \mathrm{~min}$. at $600 \mathrm{~W}$ in $10 \mathrm{mM}$ citrate buffer, $\mathrm{pH}$ 6. Slides were incubated with the biotin conjugated IgG secondary antibody and finally treated with the $\mathrm{ABC}$ staining method. The glasses were reacted with $\mathrm{DAB}$, rinsed, dehydrated in alcohol, cleared in xylene and mounted for light microscopy. Negative controls were carried out by exposition to the blocking solution instead of primary antibody.

For quantitive analyses of the results of TUNEL assays and of Ki67, CD $34^{+}$and STAT-3 immunohistologies, 10 representative $\mathrm{x} 200$ power photomicrographs in three different slides of each patient were taken with a digital camera. The positively stained cells within each photomicrograph were counted with the aid of an image processing program.

\section{Statistical analysis}

Statistical analysis was performed with paired t-test between the pre- and post-treatment values at the significance level of $\mathrm{p}<0.05$.

\section{Results}

\section{Dobesilate clears BCC lesions}

BCC were cleared and the skin showed an excellent cosmetic appearance after treatment. We report a representative patient with BCC with infiltrative clinical variant treated with topical dobesilate. Neither irritation nor adverse events were observed during and after treatment. (Figure 1A vs. 1C). Six months after treatment patients didn't show any clinical evidence of tumor recurrence.

\section{Dobesilate normalizes skin structure}

Tissue sample specimens, obtained after treatment withdrawal and stained with hematoxylin and eosin, showed a microscopic normalization of the epidermal features of the infiltrative BCC (deep epidermal hyperplasia; neoplastic keratinocyte infiltration between collagen bundles; peripheral palisading of keratinocytes protruding into the dermis; dermal clusters of neoplastic basaloid cells) (Figure 1B vs. 1D). Mitotic images were not observed in the post-treatment biopsies.

\section{Dobesilate promotes apoptosis and attenuates cell proliferation and angiogenesis in BCC}

For a thorough assessment of the observed tumor regression, we analyzed in situ apoptosis, cell proliferation and angiogenesis in tissue specimens, obtained before and after treatment. Intense inmunostaining of the proliferation marker Ki-67 was observed through entire tumor mass biopsies before treatment. Ki-67 positive cells in dobesilatetreated specimens were located mainly in the basal portion of the epidermis, suggesting a normalization of the skin homeostasis (Figure 2A vs. 2D). Quantification of Ki-67 positive cells showed a significant decrease in proliferation indices $(86.27 \pm 8.65$ vs $324 \pm 39.7 ; \mathrm{p}<0.001)$ after treatment. Tumor biopsies of patients treated with dobesilate showed a marked increase in TUNEL-positive cells in comparison with specimens obtained before treatment (Figure 2C vs. 2F). Quantification of TUNEL-stained samples showed a five-fold increase $(64.50 \pm 11.42$ vs $12.3 \pm 3.46$; $\mathrm{p}<0.001)$ in the number of TUNEL-positive cells after completion when compared with pre-treatment values. Because neovascularization is required for tumor growth, we studied the effect of dobesilate on BCC angiogenesis. For this purpose, the pre-

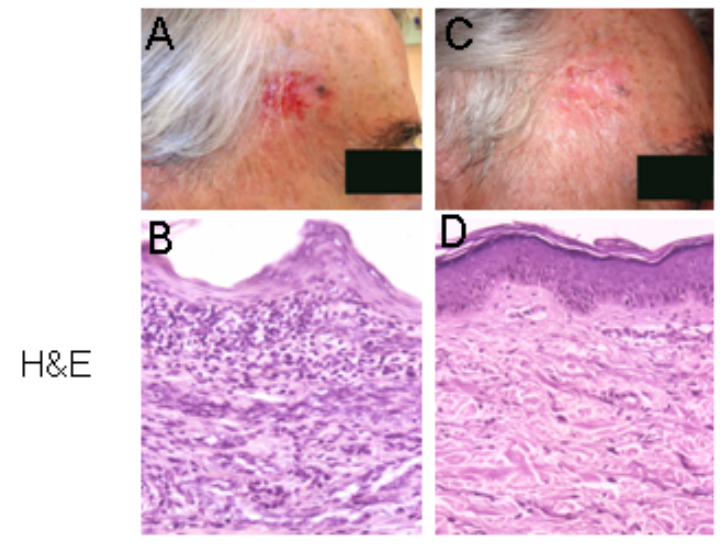

Figure 1: $A$ and $C$, macroscopic clinical features of a basal cell carcinoma before and after topical dobesilate treatment for 12 weeks, respectively. In the pre-treatment biopsy, typical features of invasive BCC that disappear after 12 weeks of topical dobesilate application are readily observed by hematoxylin and eosin staining (Panel B vs. Panel D). Original magnification B-D (x200). 
and post-treatment biopsies were immunostained against the CD34 ${ }^{+}$ endothelial cell marker. Previous to the treatment, tumors sections showed numerous large vascular lumens and branches (Figure 2B). The high vascularization in infiltrative BCC suggests the possible role of angiogenesis in determining the more aggressive biological behaviour of this type of cancer. A remarkable decrease in vascularization was observed in tumor samples after dobesilate treatment (Figure 2B vs. 2E). Quantification of tumor vessel number showed a more than twofold decrease $(8.80 \pm 3.11$ vs $19.40 \pm 6.47 ; \mathrm{p}<0.01)$ in post-treatment biopsies in comparison with the pre-treatment ones.

\section{Dobesilate inhibits STAT-3 activation}

Since cell survival, cell proliferation and angiogenesis are importantly controlled through STAT-3 activation we next analyzed its activation state in infiltrative BCC sections before and after the treatment with dobesilate, by immunohistochemical staining, using a monoclonal antibody against Tyr705-phosphorylated STAT-3. As illustrated in Figures $3 \mathrm{~A}-3 \mathrm{C}$ and $3 \mathrm{D}-3 \mathrm{~F}$, the activated state of this protein was detectable, almost exclusively, in BCC biopsies obtained before the treatment. Staining was really apparent in endothelial nuclei of neovessels, as expected (Figure 3C), but also in nuclei of keratinocytes, at the deep, medium and superficial hyperplastic epidermis. Only a weak and diffuse cytoplasmic immunostaining for activated STAT 3 could be observed in BCC biopsies after the three-month dobesilate treatment. Quantification analysis indicates that dobesilate blocks STAT-3 activation in almost $86 \%$ of tumor cells (63.28 \pm 4.47 vs 449.11 $\pm 26.68 ; \mathrm{p}<0.0001)$.

\section{Discussion}

Surgical treatment remains the standard of care for nonmelanoma skin cancer and is successful for the vast majority of patients with these tumors. The treatment of patients with metastatic or unressectable nonmelanoma skin cancer has until recently been based solely on traditional methods of chemotherapy and radiation. However, these methods have high rates of treatment failure, morbidity and mortality, and alternative treatment modalities for patients with aggressive or advanced disease are needed. In recent years, there has been

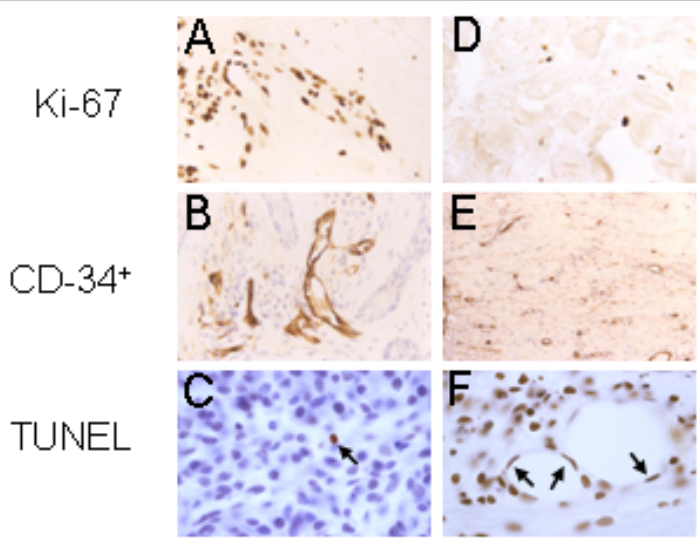

Figure 2: Numerous Ki-67 immunostained cells (brown) are selectively detected in the pre-treated specimen (Panel A vs. Panel D). CD34+ immunostaining (brown) reveals irregularly dilated vessels in pre-treated tumors (Panel B), which are absent in post-treated tumors (Panel E). A clear increase in the number of TUNEL-positive cells (brown) is observed following treatment in the hypodermis (Panel C vs. Panel F). Endothelial apoptotic nuclei in panel $\mathrm{N}$ are indicated by arrows. Panels $C$ and $F$ were counterstained with hematoxylin. Original magnification $(x 200)$.

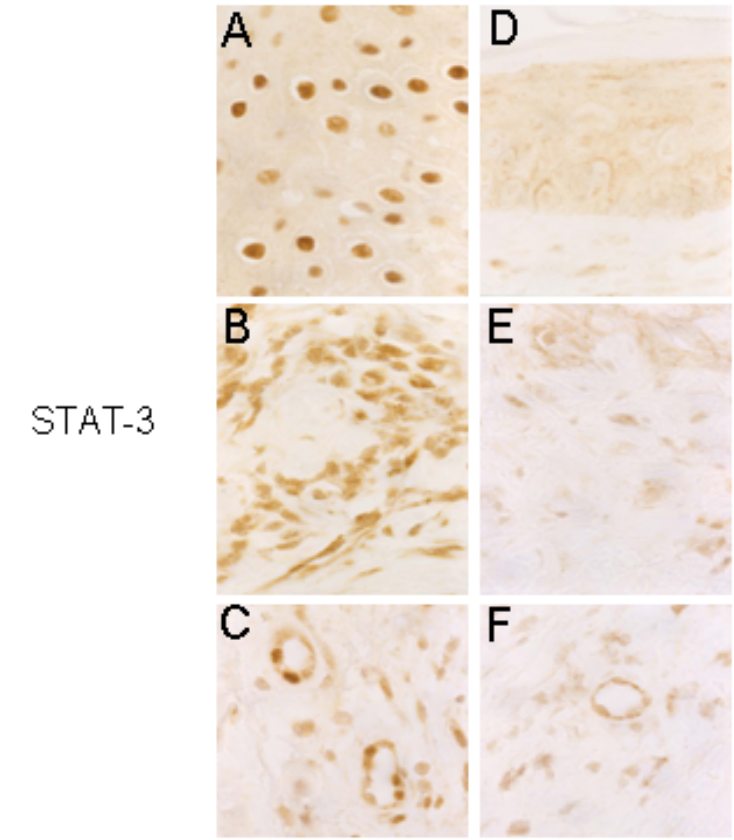

Figure 3: Immunohistochemical staining of Tyr705-phosphorylated STAT-3 in biopsies obtained before (panels $A-C$ ) and after the three month treatment (panels D-F). Sections correspond to the superficial (A and D) medium (B and $E$ ) and deep dermis ( $C$ and $F$ ), respectively. In panel $C$ two neovessels in the dermis show STAT-3 immunoreactivity in endothelial cell nuclei. All the shown pictures used to exemplify the effect of the treatment belong to the same patient.; Original magnification (x 400).

considerable interest in developing new agents to improve outcomes of these patients, focussing on novel therapeutic drugs that specifically target growth factor pathways that are upregulated in tumor cells. Such targeted therapies improve the lack of specificity of traditional cytotoxic agents, differentiating between malignant and nonmalignant cells and producing a higher therapeutic index and different toxicity profile than conventional therapies.

Constitutive activation of STAT-3 has been observed in a number of human cancers, including skin cancers [10,27]. Given that no naturally occurring STAT-3 mutations that result in constitutive activity have been identified, the persistent STAT-3 activation in tumors is likely due to differences in expression or activity of proteins that regulate STAT-3 or signalling molecules involved in the STAT-3 pathway. Potential candidates include FGF [4,6]. Given the central role of STAT-3 in multiple biological processes involved in malignant cell behaviour, extensive effort has been made to target STAT-3 and suppress its activity in cancer [28]. In this context we have demonstrated that dobesilate, an inhibitor of FGF -an upstream STAT-3 activation trigger- is able to depress the activated state levels of STAT-3. Previously we have reported that treatment of C6 glioma cells with dobesilate in vitro triggered apoptosis and growth arrest [29]. Further studies in glioma cells showed that dobesilate significantly inhibited constitutive expression of tyrosine phosphorylated STAT-3, and expression of the prosurvival proteins Bcl-xl and cyclin D1 [26]. These results support the idea that dobesilate increased apoptosis and decreased cell growth and survival in tumors, in part, by an upstream blocking of STAT-3 activation.

On the basis of the involvement of FGF/FGFR system in multiple steps of cancer development and their deregulation in a variety of 
Citation: Cuevas P, Angulo J, Cuevas-Bourdier A, Salgüero I, Giménez-Gallego G (2011) Treatment of Infiltrative Basal Cell Carcinomas by inhibiting the Fibroblast Growth Factor (FGF)-Signal Transducer and Activator of Transcription (STAT)-3 Signalling Pathways. J Cancer Sci Ther S3:003. doi:10.4172/1948-5956.S3-003

human cancers, several therapeutic strategies aiming at interfering with FGF/FGFR system activity are being developed, including small molecule tyrosine kinase inhibitors, monoclonal antibodies and FGF ligand traps. Despite their promising results in the laboratory of small molecule tyrosine kinase inhibitors against FGFR, neither of these compounds has a high probability to be successfully used in the clinic owing toxicity issues [8]. Currently, there are several ongoing efforts to generate monoclonal antibodies against FGF or FGFR. Monoclonal antibodies against FGF have shown anti-tumor activity in animal models [30]. Also monoclonal antibodies against the FGFR have shown anti-tumor effects in cancer cell lines and in mouse models [31]. These studies indicate that monoclonal antibodies targeting specific FGF or FGFR isoforms can be generated and provide proof-of-principle that therapeutic antibodies against FGF/FGFR system may have potential to be used in cancer therapy. It remains to be determined whether monoclonal antibodies targeting FGF/FGFR system will show promising results in clinical trials.

Another strategy to interfere with FGFR signalling is represented by the so-called FGF ligand traps, which sequester FGF and prevent their binding to FGFR. FGF traps may be most useful in cancers displaying FGF overexpression. The FGF trap FP-1039 (Five Prime Therapeutics) is a soluble fusion protein that prevents FGF1, FGF2 and FGF4 from binding to their respective receptors, thereby inhibiting FGFR kinase activation and therefore potentially blocking FGFRinduced proliferation and angiogenesis. This FGF trap is going to be used in Phase II clinical trials to test its activity and safety in advanced or recurrent endometrial cancers (http://Clinical Trials.gov). At this time no targeted FGF/FGFR system therapies have been developed for treatment of BCC.

Surgical excision remains the mainstay of therapy for BCC, but may not be an appropriate treatment for patients who either are not surgical candidates, very often elderly people, or refuse to undergo surgery for their skin cancer. Topical imiquimod or fluorouracil therapy may be an appropriate treatment option for those patients. Imiquimod and fluorouracil have proved efficacious in the treatment of the superficial variants of BCC. Nevertheless, up to $100 \%$ and $97 \%$ of patients applying imiquimod and fluorouracil, respectively, experience adverse events including erythema, ulceration, pruritus and pain [32]. Thus, safe and efficient topical treatments for BCC seems highly desirable.

Dobesilate is the most efficient member of a FGF inhibitor family of phenyl derivatives recently described and characterized in detail, using animal models as well as high resolution molecular and physicochemical approaches [25]. Biological assays reported in that study show that dobesilate could constitute a promising compound for developing new antitumoral therapies. We previously reported the efficacy of dobesilate in human BCC [33]. As results of these last investigations, it looks reasonably encouraging to study the mechanisms involved in the dobesilate. antitumor effect. Research regarding the involvement of angiogenesis in skin neoplasms is attracting growing attention, although these investigations have not been adequately addressed in the case of BCC. Our study is the first to compare indices of angiogenesis in infiltrative BCC, before and after topical therapy, using the panendothelial marker $\mathrm{CD} 34^{+}$which was expressed exclusively in vessellike structures Furthermore our findings are in agreement with the reported antiangiogenic effects of dobesilate, which have been shown to inhibit FGF-driven angiogenesis [25]. To confirm whether STAT-3 was activated in BCC, we performed immunohistochemical observations of the STAT-3 activation state using the appropriate antibody. Intense immunostaining for STAT-3 was observed in the nuclei of BCC cells before treatment. These findings are consistent with the repeatedly described translocation of STAT-3 subsequent to its activation, as reported in particular in skin cancers [8]. In contrast, minor staining in much fewer cells were observed in specimens obtained after dobesilate treatment. Quantitative analysis indicates that dobesilate blocks STAT3 activation in almost $86 \%$ of tumor cells. We report here that antiproliferative, pro-apoptotic and anti-angiogenic effects of topically applied dobesilate on BCC may be related to inhibition of FGF-STAT-3 signalling pathway. These congruent findings in animal models and BCC patients indicate that aberrant activation of FGF-STAT-3 pathway plays an important role in skin cancers. Taken together, these findings provide insights into molecular mechanisms of growth inhibition of BCC by dobesilate and suggest that this FGF inhibitor may be useful, when used alone or in combination with other agents, in the chemoprevention and/or treatment of BCC.

\section{Conclusion}

Targeted therapies are rationally designed to interfere with specific molecular events that are important in tumor growth, progression or survival. Targeted therapies to FGF/STAT-3 signalling pathway with anti-tumor activity is a promising approach for human cancer prevention and treatment. Inhibition of FGF/STAT-3 signalling pathway with dobesilate is a therapeutic opportunity for treating BCC and other malignancies in which FGF/STAT-3 pathway is implicated.

\section{Competing Interest}

The authors declare that they have no competing interest.

\section{Acknowledgments}

This work was supported in part by the CONSOLIDER Project CSD2009-00088 from the Spanish Ministry of Science and Innovation and by Action Medicines S.L.

\section{References}

1. Mukhtar H, Forbes PD, Ananthaswamy HN (1999) Photocarcinogenesismodels and mechanisms. Photodermatol Photoimmunol Photomed 15: 91-95.

2. Kricker A, Armstrong BK, English DR, Heenan PJ (1995) A dose-response curve for sun exposure and basal cell carcinoma. Int J Cancer 60: 482-488.

3. Bielenberg DR, Bucana CD, Sánchez R, Donawho CK, Kripke ML, et al (1998) Molecular regulation of UVB-induced cutaneous angiogenesis. J Invest Dermatol 111: 864-872.

4. Deo DD, Axelrad TW, Robert EG, Marcheselli V, Bazan NG, et al. (2002) Phosphorylation of STAT-3 in response to basic fibroblast growth factor occurs through a mechanism involving platelet-activating factor, JAK-2, and Src in human umbilical vein endothelial cells. Evidence for a dual kinase mechanism. J Biol Chem 277: 21237-21245

5. Arbiser JL, Byers HR, Cohen C, Arbeit J (2000) Altered basic fibroblast growth factor expression in common epidermal neoplasms: examination with in situ hybridization and immunohistochemistry. J Am Acad Dermatol 42: 973-977.

6. Mantovani A, Allavena P, Sica A, Balkwill F (2008) Cancer-related inflammation. Nature 454: 436-444

7. Reich NC, Lin L (2006) Tracting Stat nuclear traffic. Nat Rev Immunol 6: 602612.

8. Lin WM, Baker AC, Beroukhim R, Winckler W, Feng W, et al. (2008) Modeling genomic diversity and tumor dependency in malignant melanoma. Cancer Res 68: 664-673.

9. Brantley EC, Benveniste EN (2008) Signal transducer and activator of transcription-3: a molecular hub for signalling pathways in gliomas. Mol Cancer Res 6: 675-684.

10. Bowman T, Garcia R, Turkson J, Jove R (2000) STATs in oncogenesis Oncogene. 19: 2474-2488. 
Citation: Cuevas P, Angulo J, Cuevas-Bourdier A, Salgüero I, Giménez-Gallego G (2011) Treatment of Infiltrative Basal Cell Carcinomas by inhibiting the Fibroblast Growth Factor (FGF)-Signal Transducer and Activator of Transcription (STAT)-3 Signalling Pathways. J Cancer Sci Ther S3:003. doi:10.4172/1948-5956.S3-003

Page 5 of 5

11. Grandis JR, Drenning SD, Zeng Q, Watkins SC, Melhem MF, et al. (2000) Constitutive activation of Stat3 signaling abrogates apoptosis in squamous cell carcinogenesis in vivo. Proc Natl Acad Sci U S A 97: 4227-4232.

12. Suiqing C, Min Z, Lirong C (2005) Overexpression of phosphorylated-STAT3 correlated with the invasion and metastasis of cutaneous squamous cell carcinoma. J Dermatol 32: 354-360.

13. Syed ZA, Yin W, Hughes K, Gill JN, Shi R, et al. (2011) HGF/c-met/Stat3 signaling during skin tumor cell invasion: indications for a positive feedback loop. BMC Cancer 11: 180

14. Dechow TN, Pedranzini L, Leitch A, Leslie K, Gerald WL, et al. (2004) Requirement of matrix metalloproteinase- 9 for the transformation of human mammary epithelial cells by Stat3-C. Proc Natl Acad Sci U S A 101: 1060210607.

15. Wei D, Le X, Zheng L, Wang L, Frey JA, et al. (2003) Stat3 activation regulates the expression of vascular endothelial growth factor and human pancreatic cancer angiogenesis and metastasis. Oncogene 22: 319-329.

16. Folkman J, Shing Y (1992) Angiogenesis. J Biol Chem 267: 10931-10934.

17. Jee SH, Chu CY, Chiu HC, Huang YL, Tsai WL, et al. (2004) Interleukin-6 induced basic fibroblast growth factor-dependent angiogenesis in basal cell carcinoma cell line via JAK/STAT3 and PI3-kinase/Akt pathways. J Invest Dermatol 23: 1169-1175

18. Nahta R, Esteva FJ (2003) Bcl-2 antisense oligonucleotides: a potential novel strategy for the treatment of breast cancer. Sem Oncol 30: 143-149.

19. Wang S, Yang D, Lippman ME (2003) Targeting Bcl-2 and Bcl-XL with nonpeptidic small-molecule antagonists. Semin Oncol 30: 133-142.

20. Press MF, Lenz HJ (2007) EGFR, HER2 and VEGF pathways: validated targets for cancer treatment. Drugs 67: 2045-2075.

21. Lozano RM, Jiménez $M$, Santoro J, Rico $M$, Giménez-Gallego $G$ (1998) Solution structure of acidic fibroblast growth factor bound to 1,3 , 6-naphthalenetrisulfonate: a minimal model for the anti-tumoral action of suramins and suradistas. J Mol Biol 281: 899-915.

22. Cuevas P, Carceller F, Reimers D, Cuevas B, Lozano RM, et al. (1999) Inhibition of intra-tumoral angiogenesis and glioma growth by the fibroblast growth factor inhibitor 1,3,6-naphthalenetrisulfonate. Neurol Res 21: 481-487.
23. Cuevas P, Carceller F, Diaz D, Reimers D, Fernández M, et al. (2001) Abolished angiogenicity and tumorigenicity of rat glioma by 1 -naphthalenemonosulfonate. Neurosci Lett 308: 185-188.

24. Haritoglou C, Gerss J, Sauerland C, Kampik A, Ulbig MW; CALDIRET study group (2009) Effect of calcium dobesilate on occurrence of diabetic macular oedema (CALDIRET study): randomised, double-blind, placebo-controlled, multicentre trial. Lancet 373: 1364-1371.

25. Fernández IS, Cuevas P, Angulo J, López-Navajas P, Canales-Mayordomo A, et al. (2010) Gentisic acid, a compound associated with plant defense and a metabolite of aspirin, heads a new class of in vivo fibroblast growth factor inhibitors. J Biol Chem 285: 11714-11729.

26. Cuevas P, Díaz-González D, Sánchez I, Lozano RM, Giménez-Gallego G, et al. (2006) Dobesilate inhibits the activation of signal transducer and activator of transcription 3, and the expression of cyclin D1 and bcl- $\mathrm{XL}$ in glioma cells. Neurol Res 28: 127-130.

27. Pedranzini L, Leitch A, Bromberg J (2004) Stat3 is required for the development of skin cancer. J Clin Invest 114: 619-622.

28. Darnell JE (2005) Validating Stat3 in cancer therapy. Nat Med 11: 595-596.

29. Cuevas P, Díaz-González D, Giménez-Gallego G, Dujovny M (2005) Dihidroxy-2,5 benzenesulfonate (dobesilate) elicits growth arrest and apoptosis in glioma cells. Neurol Res 27: 797-800.

30. Shimada N, Ishii T, Imada T, Takaba K, Sasaki Y, et al. (2005) A neutralizing anti-fibroblast growth factor 8 monoclonal antibody shows potent antitumor activity against androgen-dependent mouse mammary tumors in vivo. Clin Cancer Res 11: 3897-3904.

31. Qing J, Du X, Chen Y, Chan P, Li H, et al. (2009) Antibody-based targeting of FGFR3 in bladder carcinoma and $t(4 ; 14)$-positive multiple myeloma in mice. $J$ Clin Invest 199: 1216-1229.

32. Love WE, Bernhard JD, Bordeaux JS (2009) Topical imiquimod or fluorouraci therapy for basal and squamous cell carcinoma: a systematic review. Arch Dermatol 145: 1431-1438.

33. Cuevas P, Calvo M, Angulo J, Cuevas-Bourdier A, Giménez-Gallego G (2011) Efficacy of the fibroblast growth factor inhibitor 2,5 dihydroxyphenylsulfonate in basal cell carcinoma: an histopahological and inmunohistochemical study. J Dermatolog Treat 22: 348-352.
This article was originally published in a special issue, Cancer

Chemoprevention handled by Editor(s). Dr. Rajendra Sharma, University of North Texas, USA 